\title{
Neu in der Rezensionsredaktion
}

In dieser Liste stehen alle Bücher, die die „Redaktion Buchbesprechungen“ von den Verlagen angefordert hat bzw. die von den Autoren oder Verlagen an die Redaktion geschickt wurden. Aus diesen Neuerscheinungen wählen die Herausgeber der Publizistik und die Redaktion die Bücher aus, die dann rezensiert werden.

\section{Liste der Neuerscheinungen}

Bosshart, S. (2016). Bürgerjournalismus im Web. Kollaborative Nachrichtenproduktion am Beispiel von „Wikinews“. Konstanz und München: UVK.

Engelmann, I. (2016). Gatekeeping. Baden-Baden: Nomos.

Friedmann, J. (2016). Transmediales Erzählen. Narrative Gestaltung in Literatur, Film, Graphic Novel und Game. Konstanz und München: UVK.

Jarren, O., \& Steininger, C. (Hrsg.). (2016). Journalismus jenseits von Markt und Staat. Institutionentheoretische Ansätze und Konzepte in der Publizistik- und Kommunikationswissenschaft. BadenBaden: Nomos.

Kessler, S. H. (2016). Das ist doch evident! Eine Analyse dargestellter Evidenzframes und deren Wirkung am Beispiel von TV-Wissenschaftsbeiträgen. Baden-Baden: Nomos.

Leiskau, K., Rössler, P., \& Trabert, S. (Hrsg.). (2016). Deutsche Illustrierte Presse. Journalismus und visuelle Kultur in der Weimarer Republik. Baden-Baden: Nomos.

Mahle, I. (2016). Digitale Bezahlinhalte im World Wide Web als Geschäftsmodell. Eine quantitative Befragung der ePaper-Abonnenten von Stuttgarter Zeitung und Stuttgarter Nachrichten. Baden-Baden: Nomos.

Rau, H., \& Henneke, C. (2016). Geordnete Verhältnisse?! Verflechtungsstrukturen deutscher TV-Sender. Baden-Baden: Nomos.

Simon, Y., \& Altmeppen, K.-D. (2016). Hörfunknachrichten in Deutschland. Ein Vergleich ihrer Form und ihrer Inhalte am Beispiel von ANTENNE BAYERN, BAYERN 3, HIT RADIO FFH und hr3. Baden-Baden: Nomos.

von der Lippe, B., \& Ottosen, R. (Hrsg.). (2016). Gendering War and Peace Reporting. Some Insights Some Missing Links. Göteborg: Nordicom. 